\title{
PROPOSTA DE MODELO MATEMÁTICO E ALGORITMO PARA A RECONSTRUÇÃO DA CURVA DE ELL DE SISTEMAS CONTENDO ÁLCOOIS E ÉSTERES
}

\author{
P. M. de AZEVEDO ${ }^{1,2}$, E. R. A. LIMA $^{1}$ e A. R. TÔRRES ${ }^{2}$ \\ ${ }^{1}$ Universidade do Estado do Rio de Janeiro, Programa de Pós-Graduação em Engenharia \\ Química \\ ${ }^{2}$ Universidade do Estado do Rio de Janeiro, Departamento de Química e Ambiental \\ E-mail para contato: pati_m_a@yahoo.com.br
}

RESUMO - A crescente utilização do biodiesel como combustível alternativo e complementar suscita um estudo mais detalhado destas substâncias em sistemas ternários contendo álcoois e água, por demandar conhecimento do comportamento do sistema em relação à adição de possíveis adulterantes. Neste trabalho, propôs-se um algoritmo para construção da curva de ELL correspondente, a partir de dados experimentais obtidos por titulação até a turvação (ponto de anilina). Foram utilizados os sistemas metanol/oleato de metila/água e metanol/biodiesel sintético de soja/água. Para a modelagem do ELL, foi utilizado o modelo NRTL, com parte dos parâmetros provenientes do ELV do sistema binário água e metanol ou etanol. $\mathrm{O}$ modelo foi validado com dados de equilíbrio do sistema acetona/MIBC/água. Os resultados obtidos podem ser úteis na previsão da região monofásica em sistemas ternários semelhantes ao testado, e da composição de cada uma das fases no interior da curva de ELL.

\section{INTRODUÇÃO}

Muitos pares de espécies químicas podem, em certas composições, não formar uma única fase líquida estável. Desta forma, esses sistemas dividem-se em duas ou mais fases líquidas de composições diferentes. Se estas fases estiverem em equilíbrio termodinâmico entre si, diz-se que o fenômeno é de equilíbrio líquido-líquido, ou ELL (Smith et al., 2007).

Por exemplo, ao misturar água e metil-isobutil-cetona (MIBC), a $25^{\circ} \mathrm{C}$, pode-se obter uma mistura homogênea em duas situações distintas: quando a mistura contém mais de $98 \% \mathrm{~m} / \mathrm{m}$ de água, ou quando há mais de $97,7 \% \mathrm{~m} / \mathrm{m}$ de MIBC. Em qualquer outra proporção haverá separação da mistura em duas fases distintas. Ao adicionar uma terceira substância a esta mistura bifásica, este novo composto se distribui de acordo com a sua solubilidade relativa em cada uma das fases. (Felder e Rousseau, 2005).

O comportamento de sistemas ternários parcialmente miscíveis pode ser representado em um diagrama de fases em formato de triângulo (Felder e Rousseau, 2005), no qual cada vértice representa um componente puro, seguindo em ordem decrescente de concentração em direção à aresta oposta, e estas arestas representam as misturas binárias. 


\section{9 a 22 de outubro de 2014 \\ Florianópolis/SC}

A literatura técnica é rica em resultados experimentais de diversos sistemas binários e ternários em temperaturas próximas a $25^{\circ} \mathrm{C}$ e pressão atmosférica (Poling et al., 2001). No entanto, esses dados disponíveis referem-se, em sua grande maioria, a substâncias puras e algumas moléculas de referência, como o sistema ternário acetona/MIBC/água.

Esse diagrama pode ser construído com dados experimentais por meio do "método analítico", no qual a determinação das composições pode ser feita retirando-se uma pequena alíquota de cada fase para análise de cromatografia. No caso deste trabalho, propõe-se a construção da curva de ELL a partir de dados experimentais cuja composição de apenas uma das fases é conhecida (método estático-sintético), e a reconstrução desta curva matematicamente para determinação das linhas de amarração por meio de uma modelagem matemática.

\section{MODELO TERMODINÂMICO DE G ${ }^{\mathrm{E}}-\mathrm{NRTL}$}

O modelo de NRTL (nonrandom, two-liquid), possui três parâmetros $\left(g_{i j}-g_{i i}, g_{i j}-g_{j j}\right.$ e $\left.\alpha_{i j}\right)$, ao tratar de sistemas binários. Segundo Prausnitz et al. (1999), o parâmetro $\alpha_{\mathrm{ij}}$ está relacionado com a aleatoriedade da mistura e pode ser mantido fixo e igual a 0,3 (como pode ser visto, por exemplo, em Costa, 2011) por não ter variação significativa. As equações que definem o modelo de NRTL podem ser encontradas em Prausnitz et al. (1999) e Wilding et. al. (2002) para sistemas binários. Para o caso de "n" componentes, Costa (2011) apresenta as equações expandidas do modelo NRTL.

\section{O ALGORITMO PROPOSTO}

A execução deste trabalho envolveu o cumprimento de algumas etapas, interdependentes, para alcançar o objetivo proposto. Para se obter um modelo matemático que representasse e simulasse as curvas de equilíbrio líquido-líquido dos sistemas estudados, seria necessário estimar os parâmetros do modelo NRTL para predizer propriedades dos sistemas ternários (óleo combustível, álcool e água), validar o algoritmo com um sistema de referência ( dados de ELL do sistema ternário acetona/MIBC/água) e, por fim, reconstruir as curvas dos sistemas de interesse com as respectivas linhas de amarração.

\subsection{Estratégia de Estimação dos Parâmetros}

Para facilitar a compreensão dos passos que se seguem, será adotada doravante a seguinte nomenclatura, a ser utilizada nos índices das variáveis: (A) para o álcool, (B) para o óleo, e (C) para a água.

A literatura apresenta dados suficientes e com qualidade adequada para o ELV envolvendo os álcoois (A) e a água (C) (Gmehling et al., 1977). Isso se contrasta com o fato de não existirem dados do ELL envolvendo os componentes em estudo - metanol, biodiesel metílico de soja, oleato de metila e água - ainda mais dados que descrevam as linhas de amarração. Azevedo (2013) apresenta a proposta de construção da curva de ELL com as linhas de amarração a partir dos dados experimentais de equilíbrio de sistemas ternários semelhantes ao deste trabalho.

Ao utilizar o modelo NRTL em um sistema ternário, surge a necessidade de estimar seis 
parâmetros de interação binária: três que são referentes à interação de cada composto consigo mesmo e outros três de cada composto com os outros dois componentes. Desta forma, os parâmetros obtidos por estimação dos dados de ELV para o par metanol/água podem ser utilizados nos cálculos do ELL sem perda significativa de representação. Foram obtidos 286 dados de ELV metanol/água (Gmehling et al., 1977) cuja consistência de Gibbs-Duhem estava sinalizada com "++". O software "R" foi o escolhido para o desenvolvimento do algoritmo.

\subsection{Modelo ELV}

A partir do critério de equilíbrio de igualdade de fugacidade é possível escrever a Equação 1 para um componente genérico $i$, de um sistema no qual as fases vapor e líquido estejam em equilíbrio (Smith et al., 2007).

$$
y_{i} \cdot P \cdot \varphi_{i}=x_{i} \cdot P_{i}^{v a p} \cdot \gamma_{i}
$$

Dos quais: " $y$ " é a fração molar do componente na fase vapor na pressão e temperatura do ELV; " $P$ " é a pressão do sistema; " $x$ " é a fração molar do componente na fase líquida na pressão

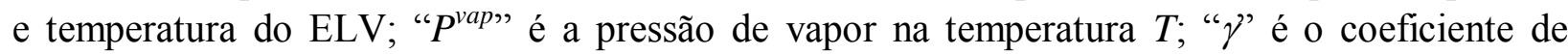
atividade; e " $\varphi$ " é o coeficiente de fugacidade.

Admitindo que os dados foram obtidos em pressões ordinárias, foi considerado que o coeficiente de fugacidade em todos os cálculos assume o valor de 1 (um), ou seja, a fase vapor foi modelada como uma mistura de gases ideais.

A pressão de vapor para os componentes foi representada em função da temperatura pela equação de Antoine. As constantes da equação de Antoine utilizadas para os álcoois estão disponíveis em Poling et al. (2001).

Para o procedimento de estimação dos parâmetros, os valores da concentração do componente na fase gasosa $(y)$ foram calculados segundo a expressão mostrada na Equação 2. A variável " $K$ " corresponde ao coeficiente de distribuição do componente entre as fases, que no caso do ELV é também chamado de coeficiente de volatilidade. Este " $K$ " foi calculado pela Equação 3.

$$
\begin{aligned}
y_{i} & =K_{i} \cdot x_{i} \\
K_{i} & =\frac{P_{i}^{v a p} \cdot \gamma_{i}}{P}
\end{aligned}
$$

\subsection{Modelo ELL}

Para o modelo de ELL foi adotada, para ambas as fases, a correção das não idealidades utilizando o coeficiente de atividade descrito pelo modelo NRTL. A Equação 4 descreve o equilíbrio de uma espécie entre as duas fases líquidas.

$$
y_{i} \cdot \gamma_{i}^{e x t}=x_{i} \cdot \gamma_{i}^{r a f}
$$


Em que: " $y$ " é a fração molar na fase extrato na temperatura do ELL; " $x$ " é a fração molar na fase rafinado, na pressão e temperatura do ELV; e " $\gamma$ " é o coeficiente de atividade do componente em cada uma das fases, calculado pelo modelo NRTL.

O modelo do ELL que foi utilizado, compatível com o algoritmo de reconstrução do diagrama ternário, foi construído tendo como incógnitas do sistema a quantidade de matéria de cada uma das espécies em cada fase. A Equação 4 foi reescrita para utilizar o coeficiente de distribuição $\mathrm{K}_{\mathrm{D}}$, definido pela Equação 5.

$$
K_{D, i}=\frac{\gamma_{i}^{r a f}}{\gamma_{i}^{\text {ext }}}
$$
6 e 7.

Os valores de $y$ e $x$ foram substituídos na Equação 4 pelos valores descritos pelas Equações

$$
\begin{aligned}
& y_{i}=\frac{n_{i}^{e x t}}{\sum_{j} n_{j}^{e x t}} \\
& x_{i}=\frac{n_{i}^{r a f}}{\sum_{j} n_{j}^{r a f}}
\end{aligned}
$$

Foram adicionadas as equações do inventário de quantidade de matéria de cada um dos componentes em cada uma das fases, Equação 8.

$$
n_{i}^{\text {total }}=n_{i}^{\text {ext }}+n_{i}^{\text {raf }}
$$

Para o sistema em questão, utilizando a notação adotada ("A" para álcool, "B" para óleo, "C" para água, "raf" para fase rafinado, "ext" para fase extrato), tem-se o sistema de equações não lineares representado pelas Equações 9.

$$
\left\{\begin{array}{l}
\frac{n_{A}^{\text {ext }}}{\sum_{j} n_{j}^{\text {ext }}}=\frac{n_{A}^{\text {raf }}}{\sum_{j} n_{j}^{\text {raf }}} \cdot K_{D, A} \\
\frac{n_{B}^{\text {ext }}}{\sum_{j} n_{j}^{\text {ext }}}=\frac{n_{B}^{\text {raf }}}{\sum_{j} n_{j}^{\text {raf }}} \cdot K_{D, B} \\
\frac{n_{C}^{\text {ext }}}{\sum_{j} n_{j}^{\text {ext }}}=\frac{n_{C}^{\text {raf }}}{\sum_{j} n_{j}^{\text {raf }}} \cdot K_{D, C} \\
n_{A}^{\text {total }}=n_{A}^{\text {ext }}+n_{A}^{\text {raf }} \\
n_{B}^{\text {total }}=n_{B}^{\text {ext }}+n_{B}^{\text {raf }} \\
n_{C}^{\text {total }}=n_{C}^{\text {ext }}+n_{C}^{\text {raf }}
\end{array}\right.
$$

Desta forma, fornecidos os valores das constantes de distribuição de cada um dos componentes e a quantidade de matéria total de cada um deles no sistema, a solução do conjunto de Equações 9 fornece a quantidade de matéria de cada um deles em cada uma das fases. As concentrações em frações mássicas são recuperadas pelas Equações 6 e 7. O sistema de equações descrito foi resolvido no ambiente " $\boldsymbol{R}$ " utilizando a função "multiroot" do pacote "rootSolve", que é um procedimento baseado no algoritmo de Newton-Rapshon, utilizando a 
matriz Jacobiana construída com um método numérico de perturbação.

\subsection{Estratégia de Construção das Linhas de Amarração das Curvas de ELL}

Os dados experimentais disponíveis não permitiam a construção das curvas de ELL com as linhas de amarração dos diagramas. A área interna das curvas fornece a composição global do sistema, e com estas linhas é possível saber qual a composição de cada uma das fases líquidas (no equilíbrio) do sistema.

Inicialmente, foram plotados os dados experimentais e, depois, foi traçada uma curva que represente este conjunto. No caso, foi utilizada uma curva "spline". Cabe esclarecer que o papel da curva spline é somente o de representar o lugar geométrico dos pontos de equilíbrio para servir como referência no cálculo da distância do ponto da solução do ELL pelo modelo e do ponto experimental. $\mathrm{O}$ valor que guiou a busca dos parâmetros foi o somatório destas distâncias.

O ponto de partida para as iterações são as concentrações correspondentes às solubilidades mútuas de água (C) em óleo (B) e de óleo em água, ou seja, as duas fases saturadas em equilíbrio sem álcool (A). A partir deste ponto incrementa-se a quantidade de matéria de " $\mathrm{A}$ " no sistema, que se divide proporcionalmente entre as duas fases, o que constitui a estimativa inicial para a solução do equilíbrio segundo o modelo descrito pelas equações do ELL.

O algoritmo começa a calcular a partir da aresta de base do diagrama ternário, de solubilidades mútuas de água e óleo, e a cada incremento em "A" é feita uma nova iteração, até que o algoritmo não consiga mais calcular o equilíbrio, pois as concentrações, nas imediações do ponto consoluto, vão ser tornando cada vez mais próximas.

Como o álcool é o componente que se distribui mais significativamente entre as duas fases, a partir da composição calculada pelo modelo do ELL, utilizam-se as frações molares dos componentes "A" e "B" $\left(x_{A}\right.$ e $\left.x_{B}\right)$ para calcular a distância destes pontos à curva "spline" traçada sobre os pontos experimentais. Esta distância entra na função objetivo do algoritmo para ser minimizada num procedimento completamente automatizado.

No final, com esta sequência de passos, o algoritmo deve ser capaz de reconstruir a curva de ELL do diagrama ternário e, assim, traçar as linhas de amarração. Desta forma, as informações necessárias a serem fornecidas ao algoritmo são: um conjunto de parâmetros para o modelo NRTL; as solubilidades mútuas de água e óleo; e um passo incremental para o aumento de quantidade de matéria de A no sistema.

\section{RESULTADOS E DISCUSSÕES}

A simplificação adotada para a estimação dos parâmetros do ELL envolve uma etapa de estimação dos parâmetros de interação entre água e metanol a partir de dados de ELV da literatura (Gmehling et al., 1977). Esta foi a etapa inicial da proposta. Estes parâmetros estão na Tabela 1.

Para testar o algoritmo proposto foi utilizado um sistema motivador constituído de acetona 
(A), MIBC (B) e água (C). Os dados deste sistema foram lidos de um diagrama ternário (Felder e Rousseau, 2005) e fornecidos ao algoritmo da mesma forma que os dados experimentais obtidos dos sistemas de interesse.

Tabela 1: Parâmetros de interação estimados do ELV para o par metanol/água

\begin{tabular}{cccc}
\hline & & Metanol & Água \\
\hline $\begin{array}{c}\text { "g" } \\
\text { (parâmetro de interação } \\
\text { do NRTL) }\end{array}$ & Metanol & 40,82 & 3568 \\
\hline
\end{tabular}

O coeficiente de distribuição foi modelado por parábolas, e os coeficientes destas constituíram os parâmetros para estimação. A Figura 1 mostra a reconstrução da curva. O diagrama está em base molar e o ponto consoluto foi o limite da construção dos estágios sucessivos de equilíbrio, correspondendo às iterações de adição da quantidade de A no sistema.

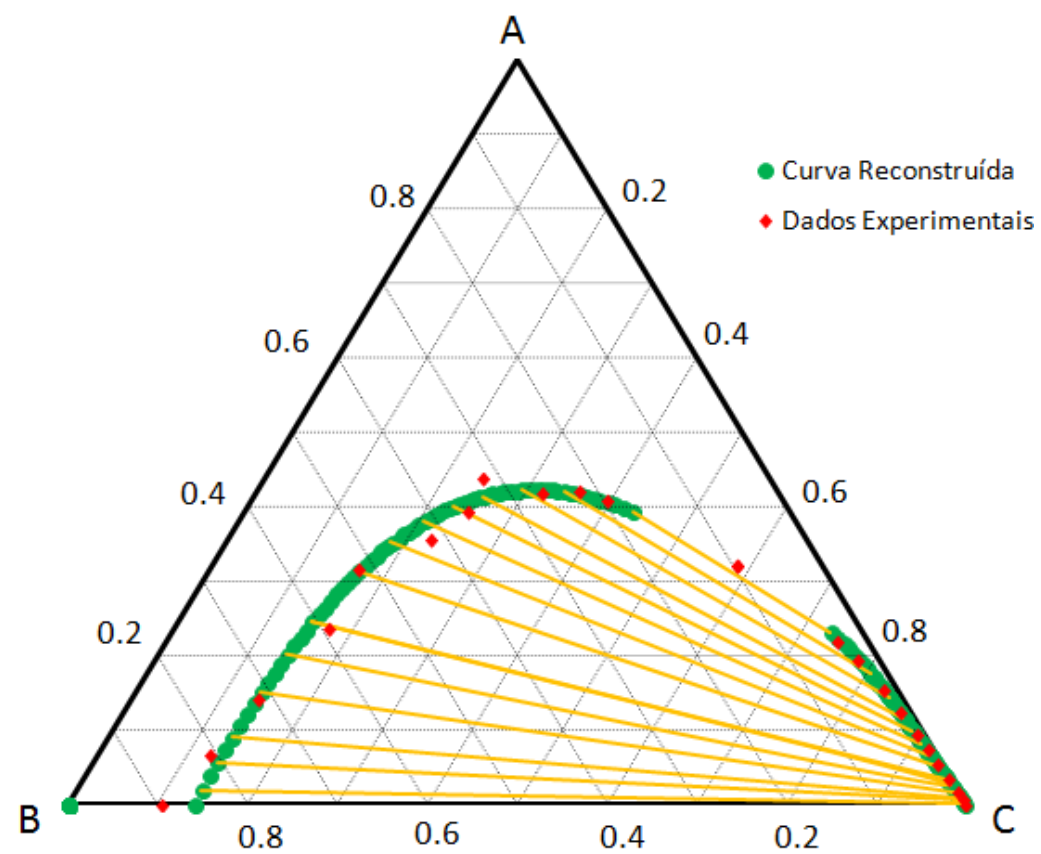

Figura 1: Diagrama ELL reconstruído para o sistema acetona (A), MIBC (B), e água (C).

O procedimento foi aplicado aos demais sistemas de interesse. A Tabela 2 contém os parâmetros utilizados nas estimativas do algoritmo proposto. E a Figura 2 apresenta estes resultados: à esquerda - metanol (A), oleato de metila (B) e água (C); e à direita - metanol (A), biodiesel sintético (B) e água (C). 
Tabela 2: Parâmetros utilizados na reconstrução das curvas de ELL do sistema metanol (A), oleato de metila / biodiesel de soja (B), e água (C).

\begin{tabular}{ccccccc}
\hline & AA & AC & CC & BA & BB & BC \\
\hline $\begin{array}{c}\text { Parâmetros utilizados na } \\
\text { reconstrução da curva da Figura 2 } \\
\text { (equerda); B = oleato de metila }\end{array}$ & 40.82 & 3568 & 4016 & 800.0 & 400.0 & 40000 \\
\hline $\begin{array}{c}\text { Parâmetros utilizados na } \\
\text { reconstrução da curva da Figura 2 } \\
\text { (direita); B = biodiesel de soja }\end{array}$ & 40.82 & 3568 & 4016 & 820.0 & 500.0 & 56000 \\
\hline
\end{tabular}

Em ambos os casos, os parâmetros do ELV não foram alterados e para o ELL foram ajustados apenas os parâmetros binários de interação do óleo (BA, BB e BC). Para o sistema contendo o oleato de metila, a sensibilidade dos parâmetros é muito elevada, e por vezes conduzem a pontos sem solução. Mas foi possível um bom ajuste do ponto consoluto. No sistema contendo o biodiesel de soja, percebe-se que a sensibilidade dos parâmetros é mais elevada que no caso anterior e, muitas vezes, também conduz a pontos sem solução. Como consequência, não foi possível estimar o ponto consoluto a partir do ajuste feito no modelo.
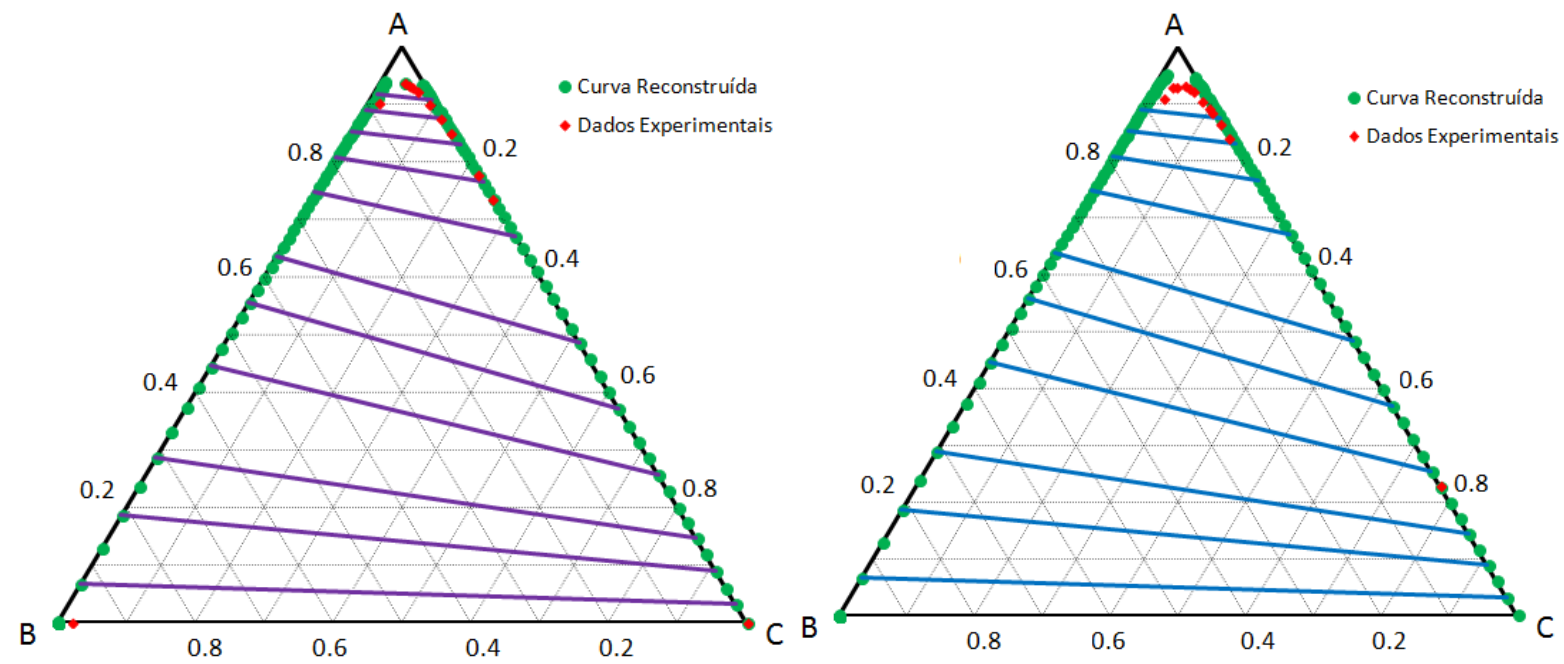

Figura 2: Diagramas ELL reconstruídos pelo algoritmo proposto com as respectivas linhas de amarração: à esquerda - metanol (A), oleato de metila (B) e água (C); e à direita - metanol (A), biodiesel sintético (B) e água (C).

\section{CONCLUSÃO}

A proposta geral foi estudar o ELL de sistemas ternários contendo oleato de metila ou biodiesel de soja pelo modelo termodinâmico NRTL com estimação dos parâmetros de interação binária, em que parte dos parâmetros foi "herdada" do ELV álcool/água, com dados da literatura. $\mathrm{O}$ método matemático proposto para reconstruir os diagramas, determinando as linhas de 
amarração, foi validado com o sistema acetona-MIBC-água, cujos dados experimentais são encontrados na literatura. Uma vez validado, o algoritmo proposto foi utilizado para reconstruir a curva de ELL do sistema metanol/oleato de metila/água, obtendo sucesso. Foi possível também encontrar a localização do ponto consoluto. A este sucesso, atribui-se também o fato do oleato de metila ser um reagente com alto grau de pureza e não conter outras substâncias com capacidade de interferir no comportamento das emulsões preparadas. Já no sistema metanol/biodiesel sintético/água, a curva foi reconstruída, mas o algoritmo não "encontrou" o ponto consoluto. Os parâmetros tinham sensibilidade maior que no caso anterior, o que dificultou a estimação.

\section{REFERÊNCIAS}

AZEVEDO, P. M.. Estudo do equilíbrio líquido-líquido e da estabilidade de sistemas envolvendo biodiesel, álcoois e água. Dissertação (Mestrado) - UERJ, 2013.

COSTA, C. T. O. G.. Equilíbrio de fases em sistemas com eletrólitos: análise de modelos de energia livre de Gibbs em excesso. Dissertação (Mestrado) - COPPE/UFRJ, Rio de Janeiro, 2011.

FELDER, R. M.; ROUSSEAU, R. W. Princípios elementares dos processos químicos. $3^{\mathrm{a}}$ ed., LTC, 2005.

GMEHLING, J.; ONKEN, U.; ARLT, W.. Vapor-Liquid Equilibrium Datacollection: Organic Hydroxyl Compounds: Alcohols. Dechema Chemistry Data Series, V.1, PT.2A. Frankfurt: Deutsche Gesellschaft Chemisches Apparatewesen, 1977.

POLING, B. E.; PRAUSNITZ, J. M.; O’CONNELL, J. P.. The Properties of gases and liquids. $5^{\mathrm{a}}$ ed., McGRAW-HILL, 2001.

PRAUSNITZ, J. M.; LICHTENTHALER, R. N.; AZEVEDO, E. G.. Molecular Thermodynamics of Fluid-phase Equilibria. $2^{\mathrm{a}}$ ed.. Prentice Hall, 1999.

SMITH, J. M.; VAN NESS, H. C.; ABBOTT, M. M. Introdução à termodinâmica da engenharia química. $7^{\mathrm{a}}$ ed., LTC, 2007.

WILDING, W. V.; ADAMS, K. L.; CARMICHAEL, A. E.; HULL, J. B.; JARMAN, T. C.; MARSHALL, T. L.. Vapor-liquid equilibrium measurements for three binary mixtures: allyl alcohol/acetonitrile, 2-Butoxyethanol/Acetic Acid, and 1-Metoxy-2-Propanol/2,3-Epoxy-1Propanol. Journal of Chemical \& Engineering, p.740-747, Washington, 2002. 